\section{The spectrum of non-classical Diamond-Blackfan anemia: a case of late beginning transfusion dependency associated to a new RPL5 mutation}

\author{
Piero Farruggia,1 Paola Quarello,2 \\ Emanuela Garelli,2 Olivia Paolicchi, 3 \\ Giovanni Battista Ruffo, 4 Liana Cuccia, ${ }^{4}$ \\ Sonia Cannella,1 Giuseppa Bruno,1 \\ Paolo D'Angelo1 \\ 1Pediatric Hematology and Oncology \\ Unit, Oncology Department, A.R.N.A.S. \\ Civico, Di Cristina and Benfratelli \\ Hospitals, Palermo; 2Pediatric \\ Department, University of Turin, Turin; \\ 3Department of Pediatric Hematology \\ and Oncology, A.O.U. Meyer Children \\ Hospital, Florence; 4 Hematology- \\ Hemoglobinopathies Unit, A.R.N.A.S. \\ Civico, Di Cristina and Benfratelli \\ Hospitals, Palermo, Italy
}

\begin{abstract}
Diamond Blackfan anemia typically presents in infants and is often associated with many kinds of malformations. Severity of anemia often needs transfusional support in the first months of life. We describe here a patient with Diamond Blackfan anemia related to a RPL5 mutation. The patient had no physical abnormalities and experienced a very late onset of transfusion dependency.
\end{abstract}

\section{Introduction}

Diamond Blackfan anemia (DBA) is a rare disease characterized primarily by normochromic macrocytic anemia and reticulocytopenia. Although anemia is the most prominent feature, the disease is also characterized by growth retardation and physical malformations, mostly craniofacial and at the extremities, but also cardiac or urogenital. These malformations are presented by almost $50 \%$ of patients. Recent advances in identifying the genotype that underlies DBA have shown involvement of genes encoding both large (RPL) and small (RPS) ribosomal subunit proteins. DBA usually presents before the age of one year. We report a case of DBA related to RPL5 mutation, without any associated major malformation, and who became transfusion dependent at the age of 12 years.

\section{Materials and Methods}

Genomic DNA was isolated from peripheral blood leukocytes, buccal epithelium cells and urinary epithelium cells using a commercial kit (Gentra Systems, Inc., Minneapolis, MN, USA).

We analyzed RPS19, RPL5 and RPL11 genes by direct sequencing of the coding exons and intron-exon boundaries. The primer sequences are available on request. Polymerase chain reaction (PCR) products were purified with the QIAquick purification kit (QIAGEN GmbH, D40724 Hilden, Germany) and sequenced on both strands with an ABI PRISM BigDye Terminator kit (Applied Biosystems, Foster City, CA, USA) on an Applied Biosystems 3100 DNA Sequencer (Applied Biosystems). When a sequence change was found, independent PCR product was sequenced to confirm the mutation. Subsequently, we sequenced DNA samples from the patient's parents to determine whether the mutation co-segregated with the DBA phenotype within the pedigree. To determine whether this sequence change was a polymorphic variation, we sequenced DNA samples from 100 Italian individuals as control and verified that it was not reported in the Single Nucleotide Polymorphism database (dbSNP at www.ncbi. nlm.nih.gov/SNP) or in the Ensembl database (www.ensembl.org). The nomenclature used to describe the sequence change is in accordance with the recommendations of the Human Genome Variation Society (http://www.hgvs. org).

All clinical samples were taken after obtaining informed consent from the proband or his relatives.

\section{Case Report}

A young male, RS, in the first months of life suffered from persistent anemia; hemoglobin levels at that time ranged between $8.0 \mathrm{~g} / \mathrm{dL}$ and $10.5 \mathrm{~g} / \mathrm{dL}$. At the age of four years, during a serious gastroenteritis, hemoglobin dropped to $6.6 \mathrm{~g} / \mathrm{dL}$ and he was transfused for the first time. At nine years of age he underwent inguinal hernia surgery under general anesthesia and no transfusion was needed. Three years later, hemoglobin level were $4.7 \mathrm{~g} / \mathrm{dL}$ and he received a blood transfusion. Since then he has been regularly transfused with packed red cells every two to three months. The anemia was macrocytic and associated with a low reticulocyte count $\left(22-25,000 / \mathrm{mm}^{3}\right)$; a mild leukopenia (WBC about $3500 / \mathrm{mm}^{3}$ ) was also present. LDH, AST, ALT, bilirubin and haptoglobin were within normal limits. There was an increase in erythropoietin levels (1600 $\mathrm{mU} / \mathrm{mL}$ ). Bone marrow aspiration showed moderate hypoplasia of the erythroid lineage
Correspondence: Piero Farruggia, Unità Operativa di Oncoematologia Pediatrica, Dipartimento di Oncologia, Padiglione 17/c, Piazza Nicola Leotta n. 4, 90127 Palermo, Italy. Tel. +39.91.6664309 - Fax +39.91.6664127. E-mail: pfarruggia@libero.it

Key words: Diamond-Blackfan anemia, transfusion-dependency, RPL5 mutation.

Acknowledgments: the authors would like to thank Prof. Frank Adamo for linguistic help and the parents' Association A.S.L.T.I.-Liberi di crescere onlus for their financial support.

Contributions: PF, conception and design of the paper, editing of the manuscript, review of the literature, final approval; PQ, EG, molecular analysis, conception and design of the paper, final approval; OP, review of the literature; GBR, LC, clinical management of the patient; SC, GB, collection of the clinical and laboratory reports; PDA, editing of the manuscript, review of the literature, final approval.

Conflict of interests: the authors report no potential conflict of interests.

Received for publication: 27 November 2011. Accepted for publication: 13 March 2012.

This work is licensed under a Creative Commons Attribution NonCommercial 3.0 License (CC BYNC 3.0).

CC Copyright P. Farruggia et al., 2012

Licensee PAGEPress, Italy

Pediatric Reports 2012; 4:e25

doi:10.4081/pr.2012.e25

with myeloid and megakaryocytic cells of a normal aspect and without any signs of dysplasia. In vitro erythroid colony formation was drastically reduced, whereas myeloid and megakaryocytic colony were normal. At that time (2002), it was impossible to make a definite diagnosis. Hemoglobin electrophoresis excluded thalassemia. Direct antiglobulin test (DAT) and indirect antiglobulin tests were negative, pyruvate kinase and glucose-6-phosphate dehydrogenase were in the normal range, the karyotype was normal and, finally, screening for autoimmunity (ANA, ENA, anti-DNA, AMA, ASMA, antigliadin antibodies), Parvovirus PCR, Perls' reaction in the bone marrow smear and diepoxybutane test were all negative. No sequence variations were detected in RPS19.

In the aftermath, other ribosomal genes were analyzed and a new single nucleotide deletion in exon 4 of RPL5 was discovered (c.283delT;p.Tyr95MetfsX31). According to the description of the majority of DBA patients, 1 this was a de novo mutation not found in his parents. The clinical course suggested a mosaicism. To exclude this, we sequenced the 
DNA extracted from buccal and urinary epithelium and confirmed the mutation.

An increase of erythrocyte adenosine deaminase activity is present in the majority of patients. ${ }^{2}$ However, because of the patient's transfusion dependency, it was impossible to verify any increase in this. A trial of prednisone therapy proved to be partially effective only at a dosage of $3 \mathrm{mg} / \mathrm{kg} /$ day. Now the patient is following a transfusion regimen and iron chelation with deferasirox. He is short in stature and is well proportioned without any physical abnormalities or mental retardation.

\section{Discussion}

DBA is a clinically heterogeneous lineageselective bone marrow failure syndrome. In addition to malformations and anemia, the disease is associated with predisposition to cancer, particularly acute myeloid leukemia and osteogenic sarcoma. ${ }^{3}$

Diagnostic criteria have recently been established at an International Consensus Conference (Table 1). ${ }^{1}$ A diagnosis of classical DBA is made in the presence of all the diagnostic criteria and a diagnosis of non-classical DBA in the presence of: i) 3 diagnostic criteria and one major supporting criterion or 2 minor criteria; ii) 2 diagnostic criteria and 3 minor supporting criteria; iii) 2 major supporting criteria, even in the absence of diagnostic criteria.

Age under one year is a diagnostic criterion and it is a common experience that DBA is a disease of the first weeks or months of life. It is known that anemia in DBA may be transient but there are reports in the literature of a few cases of DBA that were first recognized in late childhood or in adulthood.4,5 In our patient, even though he had been anemic since the first months of life it had been possible to maintain satisfactory clinical condition without transfusion. After the first year of life, the hemoglobin level increased and remained stable $(9-10 \mathrm{~g} / \mathrm{dL})$ till the patient's $12^{\text {th }}$ year. Anemia only worsened at the age of 12 years and since then, regular red blood cell transfusions have been required.

About half of the affected patients have been found to have a heterozygous mutation in a gene encoding a small (RPS7, RPS10, RPS17, RPS19, RPS24, RPS26) or large (RPL5, RPL11, $R P L 35 a)$ ribosomal subunit-associated protein. ${ }^{1,6}$ RPL5 mutations are reported in approximately $7-21 \%$ of DBA patients. ${ }^{7,8}$ Birth defects, present in approximately $40-55 \%$ of DBA patients, 7,8 are more often associated with mutations in RPL11 and, above all, RPL5.7-10 Among RPL5 patients, anomalies of thumb and cleft lip and/or palate appear to be the most frequent. ${ }^{7-9}$ This was not the case of our patient; measurements at birth were small for gestational age as has been reported in Western patients but not in Japan.7,8 Remarkably, all the mutations reported in the Japanese population regard exon 8 whereas in Western countries they have been described in exons 1,2, 3, 5 and 6 and in introns $1,2,3,4,5$ and $6.5,9$ Furthermore, to our knowledge there have only been 5 other published cases of RPL5 mutation found in exon 4 for which clinical details are available. ${ }^{5}$ In fact, no medical information is available for another 2 cases. 10 So, including our patient, there have been 6 evaluable reported cases of RPL5 mutations in exon 4 and one other case in whom the mutation (in intron 4) resulted in a complete deletion of exon $4 ;^{9}$ among these 7 cases (Table 2), 3 out of 6 evaluable patients were characterized by a delayed onset, one by a spontaneous remission and 3 by absence of birth defects. Remarkably, in some of the larger series, ${ }^{8,9}$ malformations were found

Table 1. Diagnosis of Diamond-Blackfan anemia.

Diagnostic criteria
Age less than 1 year
Macrocytic anemia
Reticulocytopenia
Normal marrow cellularity with a paucity of erythroid precursors
Supporting criteria
Major
- RPS19 mutation described in classical DBA
Minor $\quad$ Positive family history
- Elevated erythrocyte adenosine deaminase activity
- Elevated fetal hemoglobin
- No evidence of another inherited bone marrow failure syndrome

Table 2. Diamond-Blackfan anemia patients with mutation in exon 4 or intron 4.

\begin{tabular}{|c|c|c|c|c|c|c|c|c|}
\hline Patients & Inheritance & Exon/Intron & Mutation & Protein & Gender & Malformations & Age at Dx & Therapy \\
\hline $\begin{array}{l}\text { Gazda et al. (2008) } \\
\text { Am J Hum Genet 83(6), 769-80 }\end{array}$ & De novo & Exon 4 & c. $228 \mathrm{C} / \mathrm{A}$ & p.Cys76X & $\mathrm{F}$ & Yes & $10 \mathrm{mos}$ & HSCT \\
\hline $\begin{array}{l}\text { Gazda et al. (2008) } \\
\text { Am J Hum Genet 83(6), 769-80 }\end{array}$ & Familial & Exon 4 & c.235_236 insT & p.Tyr79LeufsX34 & $\mathrm{F}$ & Yes & $11 \mathrm{yrs}$ & RBC trx \\
\hline $\begin{array}{l}\text { Gazda et al. (2008) } \\
\text { Am J Hum Genet 83(6), 769-80 }\end{array}$ & Familial & Exon 4 & c.235_236 insT & p.Tyr79LeufsX34 & $\mathrm{F}$ & No & $55 \mathrm{yrs}$ & $\mathrm{RBC}$ trx \\
\hline $\begin{array}{l}\text { Gazda et al. (2008) } \\
\text { Am J Hum Genet 83(6), 769-80 }\end{array}$ & Familial & Exon 4 & c.235_236 insT & p.Tyr79LeufsX34 & $\mathrm{F}$ & Yes & $12 \mathrm{mos}$ & RBC trx \\
\hline $\begin{array}{l}\text { Gazda et al. (2008) } \\
\text { Am J Hum Genet 83(6), 769-80 }\end{array}$ & Familial & Exon 4 & c.235_236 insT & p.Tyr79LeufsX34 & $\mathrm{F}$ & No & NA & RBC trx \\
\hline $\begin{array}{l}\text { Quarello et al. (2009) } \\
\text { Haematologica } 95(2), 206-213\end{array}$ & De novo & Intron 4 & c. $324+5 \mathrm{G}>\mathrm{T}$ & p. $0 ?$ & $\mathrm{~F}$ & Yes & $1 \mathrm{mo}^{*}$ & No therapy \\
\hline Present report & De novo & Exon 4 & c.283delT & p.Tyr95MetfsX31 & M & No & $12 \mathrm{yrs}^{* *}$ & RBC trx \\
\hline
\end{tabular}

Dx: diagnosis; F=female; mos: months; HSCT=Hematopoietic stem cell transplantation; yrs: years; M=male; NA=not available; RBC trx=red blood cells transfusion.

${ }^{*}$ Spontaneous remission after one RBC trx. ${ }^{* *}$ Anemia with transfusion dependency diagnosed at age 12 , DBA not diagnosed until years later. 
in $83-100 \%$ of $R P L 5$-mutated patients. To our knowledge there has been only one other case of RPL5 mutation in exons other than 4 (exon 5 ) whose age at presentation was early adulthood (20 years). ${ }^{5}$ In spite of this, because of the paucity of cases, not enough information is available to confirm that RPL5-DBA patients with exon 4 mutations have a milder phenotype than other RPL5-DBA patients.

Corticosteroids and transfusions are the mainstays of DBA treatment and RPL5 mutation does not appear to be predictive of response. ${ }^{7,9}$ Our patient was unresponsive to corticosteroids at standard doses and he is receiving red blood cell transfusions according to thalassemia regimens. Given the fact that he is no longer a small child and that he enjoys a good quality of life, allogeneic bone marrow transplantation has not been considered.

\section{Conclusions}

In conclusion, we believe that a diagnosis of DBA can also be considered in cases of anemia that do not present in early childhood. It is pos- sible that DBA-affected individuals with an RPL5 mutation in exon 4 may present a less severe phenotype than other RLP5-DBA patients.

\section{References}

1. Vlachos A, Ball S, Dahl N, et al. Diagnosing and treating Diamond Blackfan anaemia: results of an international clinical consensus conference. Br J Haematol 2008;142: 859-76.

2. Glader BE, Backer K, Diamond LK. Elevated erythrocyte adenosine deaminase activity in congenital hypoplastic anemia. N Engl J Med 1983;309:1486-90.

3. Vlachos A, Klein GW, Lipton JM. The Diamond Blackfan anemia registry: tool for investigating the epidemiology and biology of Diamond-Blackfan anemia. J Pediatr Hematol Oncol 2001;23;377-82.

4. Balaban EP, Buchanan GR, Graham M, et al. Diamond-Blackfan syndrome in adult patients. Am J Med 1985;78:533-8.

5. Gazda HT, Sheen MR, Vlachos A, et al. Ribosomal protein L5 and L11 mutations are associated with cleft palate and abnormal thumbs in Diamond-Blackfan anemia patients. Am J Hum Genet 2008;83:769-80.

6. Farrar JE, Dahl N. Untangling the phenotypic heterogeneity of Diamond Blackfan anemia. Semin Hematol 2011;48:124-35.

7. Konno Y, Toki T, Tandai S et al. Mutations in the ribosomal protein genes in Japanese patients with Diamond-Blackfan anemia. Haematologica 2010;95:1293-9.

8. Cmejla R, Cmejlova J, Handrkova H, et al. Identification of mutations in the ribosomal protein L5 (RPL5) and ribosomal protein L11 (RPL11) genes in Czech patients with Diamond-Blackfan anemia. Hum Mutat 2009;30:321-7.

9. Quarello P, Garelli E, Carando A, et al. Diamond-Blackfan anemia: genotype-phenotype correlations in Italian patients with RPL5 and RPL11 mutations. Haematologica 2010;95:206-13.

10. Boria I, Garelli E, Gazda HT, et al. The ribosomal basis of Diamond-Blackfan Anemia: mutation and database update. Hum Mutat 2010;31:1269-79. 